\title{
COMPREENSÃO DE EXPRESSÕES IDIOMÁTICAS DA LÍNGUA PORTUGUESA COMO L2: EVIDÊNCIAS DE PROTOCOLOS VERBAIS
}

\author{
Alessandra Baldo* \\ Universidade Federal de Pelotas \\ Pelotas, Rio Grande do Sul, Brasil
}

\begin{abstract}
Resumo: Este artigo relata um estudo cujo objetivo foi investigar em que medida os processos cognitivos utilizados por cinco falantes de português como L2 para compreender dez expressões idiomáticas (EIs) de forma descontextualizada seriam similares aos utilizados para a compreensão das mesmas expressões em contexto. A análise dos dados, coletados por meio de protocolos verbais retrospectivos e de pausa, revelou que o uso de analogia e associações a partir de conhecimentos prévios foi a estratégia preferencial para as EIs descontextualizadas, enquanto o uso de indícios microtextuais foi a mais empregada nas EIs em contexto. Além disso, os dados apontaram para uma aproximação apenas parcial dos processos cognitivos empregados pelos participantes nos dois contextos de atribuição de significado a novas EIs. Os resultados são avaliados com base em Liontas (2001, 2002), Polónia (2009) e Sadeghi, Dastjerd e Ketabi (2010).
\end{abstract}

Palavras-chave: Expressões idiomáticas. Processos inferenciais. Português como L2.

1 INTRODUÇÃO

O ensino de expressões idiomáticas (EIs) nas aulas de português como língua estrangeira (L2) configura-se como um recurso privilegiado para fomentar a interculturalidade (ALVAREZ, 2000; ALVAREZ e MACHADO, 2010; RIVA e CAMACHO, 2010, SILVA, 2011), o que pode ser atestado pelos estudos voltados a apresentar a melhor forma de ensiná-las, como também pela diversidade de materiais didáticos atualmente disponíveis para seu ensino.

Contudo, o que se observa de modo menos significativo nessa área são estudos sobre os processos cognitivos empregados pelos aprendizes para a compreensão dessas expressões, e é nessa lacuna que o estudo aqui relatado se insere. De natureza qualitativa e utilizando a técnica de protocolos verbais, o foco está nas estratégias inferenciais de cinco estudantes de português como L2 nas suas tentativas de buscarem o significado de EIs desconhecidas.

Mais detalhadamente, os objetivos do estudo foram três: (i) avaliar as estratégias cognitivas empregadas por cinco aprendizes de português como L2 para inferir o significado de EIs quando apresentadas de modo descontextualizado; (ii) avaliar as estratégias cognitivas empregadas pelos mesmos aprendizes para inferir o significado dessas expressões quando apresentadas em um contexto específico; (iii) avaliar em que

\footnotetext{
* Doutora em Letras pela Pontifícia Universidade Católica do Rio Grande do Sul (PUCRS). Professora Adjunta do Centro de Letras e Comunicação. Email: alessabaldo@gmail.com.
} 
medida seria possível aproximar as estratégias utilizadas pelos sujeitos nos dois contextos de atribuição de significado a novas EIs.

Foram lançadas três questões norteadoras, em conformidade com os objetivos: (i) quais estratégias são empregadas para realizar as inferências das EIs sem contexto; (ii) quais estratégias são utilizadas para as inferências contextualizadas?; (iii) há estratégias inferenciais em comum entre as EIs descontextualizadas e as contextualizadas?

O artigo está organizado em cinco partes. As duas primeiras são reservadas aos preceitos teóricos do estudo. Na primeira parte, as relações entre ensino-aprendizagem de EIs e de L2 são brevemente resgatadas. Na segunda - EIs e estratégias cognitivas são revistos conceitos e pesquisas-chave para o estudo aqui descrito. A metodologia é descrita na terceira parte, seguida pela descrição e análise dos dados obtidos por meio dos protocolos verbais. A quinta e última parte é reservada a uma reflexão com base nos resultados viabilizados pelos dados, e também a uma proposta de continuidade da pesquisa.

\title{
2 EIS E ENSINO-APRENDIZAGEM DE L2
}

Definidas como "uma das manifestações mais relevantes das potencialidades criadoras de uma língua" (ORTIZ ALVAREZ, 2007, p.03), devido à riqueza de suas imagens, originalidade de suas metáforas e variedade e maleabilidade em suas formas estruturais, as expressões idiomáticas (EIs) têm figurado como tema de um número considerável de estudos relativos ao ensino-aprendizagem de línguas estrangeiras. Entre os enfoques dos estudos, a relação indissociável entre ensino de EIs e ensino de cultura tem sido dos mais constantes. ${ }^{1}$

De fato, entre os diferentes tipos de palavras e unidades fraseológicas que compõem o léxico de uma língua, entendemos que as EIs são as que possibilitam um contato maior do aprendiz com os diferentes usos da L2 - ou seja, literais e figurados -, favorecendo um maior conhecimento da cultura da língua-alvo. Como esse conhecimento é imprescindível para o estabelecimento de possíveis relações interculturais entre a L1 e a L2, as EIs são fenômenos linguísticos privilegiados para o estabelecimento de tais relações devido a características como a expressividade, a conotação, a convencionalidade, em especial nos níveis semântico e pragmático, e a indecomponibilidade (TAGNIN, 2005).

Além desse, Kramsch (1999, p. 243) salienta outro aspecto positivo que a aquisição de EIs proporciona aos aprendizes de uma L2, ou seja, a satisfação de sentir que estão obtendo domínio de uma língua que, inicialmente, lhes era desconhecida.

Nas palavras da autora:

\begin{abstract}
A emoção de conseguir usar formas de discurso que são reservadas somente para falantes nativos, como gírias e expressões altamente idiomáticas, é uma das formas através da qual os aprendizes ganham poder dentro de um sistema que, por sua natureza, os lembra quão impotentes eles realmente são. (KRAMSCH, 1993, p.243, tradução nossa)
\end{abstract}

\footnotetext{
${ }^{1}$ A título de ilustração, citamos Pedro, 2007; Ortiz Alvarez, 2007; Polónia, 2009; Silva, 2011.
} 
Assim, não somente o ensino das EIs é relevante pelo conhecimento linguístico e cultural que revela ao aprendiz, mas também pela oportunidade que lhe oferece de sentir-se confiante no seu processo de aprendizagem de L2. O conhecimento de EIs, desse modo, conseguiria tornar mais próximo do aprendiz o novo sistema linguístico que está sendo construído, e essa proximidade, sabemos, é um dos fatores determinantes para a aprendizagem bem-sucedida de uma L2.

Além disso, a relativização dos valores morais é outro aspecto do ensino de L2 que encontra terreno fértil para o desenvolvimento com a apresentação de EIs aos aprendizes. O processo de refletir sobre as possíveis razões para EIs não encontrarem equivalentes em sua L1 e, por outro lado, algumas serem bastante semelhantes, como também sobre as motivações para a atribuição de determinado significado a uma dada expressão, somente pode ocorrer de fato na medida em que o aprendiz avaliar diferenças e semelhanças culturais entre as duas línguas. ${ }^{2}$ A compreensão de Gibbs (1994) sobre as condições de uso das EIs é, nesse contexto, bastante esclarecedora:

\begin{abstract}
As condições pragmáticas de uso das expressões idiomáticas não são arbitrárias ou, a rigor, uma questão de convenção, mas podem ser diretamente motivadas pelo entendimento das estruturas conceituais que subjazem às expressões idiomáticas e pela conceituação dos eventos e conceitos em diferentes situações discursivas. (GIBBS, 1994, p. 302, tradução nossa)
\end{abstract}

A não-arbitrariedade do uso das EIs também é sublinhada por Silva (2011), ao analisar EIs que encerram relações metonímicas à luz da teoria de Lakoff e Johnson (1980). De acordo com a autora, as pessoas percebem e categorizam o mundo tendo como critério noções de semelhança e diferença, a partir de conhecimentos já adquiridos.

Como veremos adiante, os resultados deste estudo confirmam a noção de que esse processo de categorização responde, de fato, por uma das estratégias de uso mais frequente por aprendizes de uma L2 ao realizarem as inferências das EIs.

\title{
3 EIS E ESTRATÉGIAS COGNITIVAS
}

Iniciamos esta seção retomando e expandindo a informação que abre este manuscrito: enquanto há muitos estudos de EIs em L1 ou em L2 voltados (i) a formas adequadas de ensiná-las e (ii) a sua caracterização, com consequente distinção entre essas e outros fenômenos fraseológicos das línguas naturais, o mesmo não ocorre com pesquisas com foco nas estratégias de processamento das EIs por aprendizes de L2.

Entre os estudos voltados especificamente a esse tema, retomaremos os trabalhos de Cooper (1999), Liontas (2001, 2002), Bulut e Çelik-Yazici (2004), Polónia (2009) e

\footnotetext{
${ }^{2}$ Pela abordagem interculturalista de ensino de língua, o papel do professor de LA, nesse contexto, é o de "colocar em prática a ideia de que é possível tornar familiar o que é pouco conhecido, ou seja, aquilo que pertence a uma cultura que não a sua própria", questionando, ao mesmo tempo, o que lhe parece familiar, ou seja, "elementos da cultura de origem que devem ser apreciados sob novos pontos de vista". (PARANÁ; ALMEIDA, 2005, p. 72).
} 
Sadeghi, Dastjerdi e Ketabi (2010). Antes disso, apresentamos a pesquisa de Nassaji (2003), a única que difere das anteriores na medida em que analisa as estratégias inferenciais empregadas por aprendizes de L2 para a compreensão de qualquer categoria de palavra, e não somente de EIs .

Nassaji buscou responder duas questões em seu estudo: (i) como estudantes de nível intermediário de inglês como L2 inferem o significado de novas palavras?; (ii) que estratégias e fontes de conhecimento são empregadas, ${ }^{3}$ e em que medida? Como sujeitos, contou com 21 aprendizes adultos, de diferentes L1s, cuja tarefa era verificar o significado de palavras novas apresentadas em um texto.

Através da análise dos protocolos verbais dos participantes, o autor chegou às seguintes conclusões: (i) a fonte de conhecimento mais usada foi o conhecimento de mundo/enciclopédico, e a menos, o conhecimento gramatical. Como implicação natural, sugere que é a relação semântica que se sobrepõe ao conhecimento gramatical na inferência lexical; (ii) a repetição foi a estratégia mais empregada, o que não seria surpreendente na medida em que ela pode auxiliar na compreensão e reflexão sobre conteúdo; (iii) a repetição da seção na qual a palavra se encontrava foi responsável por um maior número de inferências lexicais apropriadas do que unicamente a repetição da palavra, e isso estaria relacionado ao papel da primeira estratégia, de auxiliar o aprendiz a relacionar a palavra com a frase na qual ela ocorre e a usar os indícios potenciais disponíveis nesses contextos; (iv) as estratégias de verificação e autoavaliação foram mais relacionadas com sucesso inferencial do que outras estratégias, o que contaria como evidência do papel das estratégias metacognitivas em auxiliar aprendizes a avaliar a exatidão de suas inferências e revisá-la e/ou reavaliá-las com base na informação fornecida no contexto mais amplo;

Especificamente sobre o processamento de EIs no contexto de ensinoaprendizagem de L2, um dos estudos mais citados é o de Cooper (1999), sobre as estratégias empregadas por dezoito universitários aprendizes de inglês como L2 para descobrir o significado de vinte EIs de uso comum. A conclusão principal do autor foi de que a inferência pelo contexto foi a mais empregada, com $28 \%$ do total. Além dessa, outras duas estratégias foram utilizadas de modo significativo: análise do idioma, com $24 \%$ do total, e uso do significado literal, com $19 \%$. Os aprendizes também se valeram de estratégias de outra natureza, mas de modo menos expressivo: enquanto a solicitação de informação foi responsável por $8 \%$ do total de estratégias, a paráfrase ou repetição, a utilização de conhecimento prévio e o emprego da L1 tiveram, cada uma, 7\% do total.

A exemplo de Cooper, a importância do contexto como estratégia para identificação de EIs por aprendizes de L2 também é enfatizada por Liontas (2001, 2002) - e, como veremos, nos demais estudos relatados nesta seção. Na pesquisa de 2001, o autor analisa os processos cognitivos para percepção e compreensão de EIs por onze americanos aprendizes de grego como L2, e também investiga em que medida o

\footnotetext{
${ }^{3}$ Ao invés de utilizar somente o termo estratégias, de uso corrente na literatura, o autor optou por subdividir os recursos cognitivos em estratégias e fontes de conhecimento. Do primeiro grupo fazem parte a releitura, repetição da palavra ou seção do texto, verificação, autoquestionamento, análise, monitoramento e analogia. Do segundo grupo, o conhecimento gramatical, morfológico, da L1, de mundo/enciclopédico e discursivo.
} 
contexto afeta a compreensão dessas EIs. Liontas (2001, p. 10) concluiu que a maioria dos aprendizes foi capaz de inferir o significado apropriado das expressões "inseridas em parágrafos e diálogos autênticos, valendo-se de uma variedade de estratégias de leitura e princípios pragmáticos de comunicação"(tradução nossa). A grande maioria dos participantes empregou as seguintes estratégias: significado literal das EIs (23.8 \%), tradução tanto da L2-L1 como da L1-L2 (17\%), estrutura sintática e semântica das EIs (11.4\%), conhecimento prévio das funções das EIs em contexto $(8.8 \%)$, e o contexto em torno das EIs (8\%). Esses dados, para Liontas, fornecem evidência robusta de que aprendizes de L2 processam os significados literal e idiomático das EIs separadamente, produzindo duas interpretações alternativas, com o significado idiomático estando disponível somente depois de o significado literal ter sido considerado e descartado. Essa evidência, assim, alerta o autor, contradiz as teorias psicolinguísticas que definem as EIs como uma única unidade armazenada no léxico mental (GIBBS, 1984; CORNELL, 1999), mostrando que, para o aprendiz de L2, o processo pode ser exatamente o oposto.

$\mathrm{Na}$ pesquisa de 2002, Liontas mantém basicamente os mesmos objetivos da precedente, mas com um número maior de participantes - 53 ao todo -, os quais possuíam como L1 espanhol, francês ou alemão. Com relação às estratégias empregadas para compreender as EIs em inglês como L2, todas pertencentes à categoria de verbos frasais, o autor concluiu que tradução, adivinhação (guessing) e uso do contexto foram as mais importantes. Além disso, o estudo revelou que o grau de opacidade entre a L1 e a L2, conhecimento de vocabulário e o significado literal das EIs afetam a transferência do conhecimento idiomático de modo significativo.

Bulut e Çelik-Yazici (2004) já partem do pressuposto de que tanto o contexto como o significado literal afetam a compreensão das EIs na L2, e desenham um estudo com o objetivo de validar a hipótese de que o contexto teria maior impacto na compreensão das novas EIs, em detrimento do significado literal. Além disso, os autores também hipotetizaram que as EIs na L2 com equivalentes na L1 seriam mais facilmente compreendidas do que as demais. Tendo como participantes 18 professores turcos de inglês como L2, tiveram confirmada a primeira hipótese do estudo, e apenas parcialmente confirmada a segunda.

O uso do contexto para a interpretação do significado foi a estratégia mais usada, e a única estratégia estatisticamente significativa. O significado literal das EIs e o conhecimento prévio também foram empregados, mas em uma proporção bem menor. Já com relação às EIs com equivalentes na L1, ainda que a aproximação com a L1 tenha sido a estratégia de escolha na maioria dos casos, alguns sujeitos as avaliaram como falsos cognatos, a partir de sua experiência como professores de inglês, e optaram por empregar o contexto como estratégia para compreendê-las.

Em Sadeghi, Dastjerdi e Ketabi (2010), o papel do contexto na compreensão de EIs na L2 é novamente avaliado em 60 estudantes persas de inglês como L2, divididos em três grupos de proficiência - básico, intermediário e avançado. Além disso, a influência do grau de opacidade entre as EIs na L1 e na L2 e do conhecimento dos lexemas presentes nas EI também foi avaliada. Os principais resultados, já relatados por estudos anteriores, foram: o contexto tem um papel importante na construção do 
significado idiomático; quanto maior a opacidade da EI, mais difícil a sua interpretação; o tratamento das EIs como conjuntos de itens lexicais em separado, independentemente do sistema cultural e conceptual humano, leva à transferência negativa. Além disso, os autores argumentam que os dados, especialmente os obtidos pelos protocolos verbais retrospectivos, evidenciaram que os significados idiomáticos e literais das EIs são processados separadamente por aprendizes de L2. Para os autores, isso mostra que o sentido idiomático torna-se disponível somente depois que a interpretação literal é considerada e rejeitada.

Conforme colocado no início da seção, o estudo de Polónia (2009) é o único, entre os descritos anteriormente, a ter participantes com a mesma L2 - ou seja, português do que o apresentado aqui. A autora teve como objetivos investigar se aprendizes de L2 seriam capazes de (i) reconhecer EIs descontextualizadas e/ou implicitamente, (ii) realizar analogias com sua L1 e (iii) aprender o significado das EIs quando apresentadas em crônicas e textos publicitários. A partir da aplicação de uma série de materiais didáticos com EIs diversas, a pesquisadora chegou às seguintes conclusões: 0 reconhecimento das EIs fora de contexto era bastante difícil para os aprendizes, a menos que já as tivessem ouvido e/ou lido anteriormente; analogias com a L1 como forma de aprender o significado da EI na L2 eram feitas com frequência, mas em geral somente quando havia equivalência de sentido alinhada a uma estrutura semânticosintática muito semelhante entre a expressão na L1 e a expressão na L2; os aprendizes se valeram ao máximo do contexto - crônicas e textos do gênero publicitário - para inferir o significado das EIs, mas isso não garantiu uma compreensão adequada em todos os casos, o que levou Polónia a afirmar que "nem sempre um único contexto é suficiente para que os alunos decodifiquem os significados das EIs" (2009, p. 69).

\section{METODOLOGIA}

A fim de ser possível responder às questões colocadas pelo estudo, foram selecionados cinco alunos intercambistas, matriculados no Nível I do curso de Português para Estrangeiros da Universidade Federal de Pelotas no segundo semestre de 2012. O curso teve duração de quatro meses, com aulas ministradas três vezes por semana, totalizando 120 horas/aula. Atividades de leitura, escrita, conversação e compreensão auditiva foram desenvolvidas sistematicamente durante as aulas.

A oferta regular de cursos de português como L2 iniciou na Universidade no ano de 2011, visando a atender as demandas de um número crescente de intercambistas estrangeiros que chegam à instituição com pouco - ou mesmo nenhum - conhecimento da língua.

A seleção dos sujeitos teve como critério o teste de habilidade linguística em Português como L2 realizado no final do curso: os alunos com um percentual de $80 \%$ ou mais de acertos foram convidados a participar do estudo. Entre os cinco voluntários, três deles eram falantes de espanhol como língua materna, um, de chinês, e o último, de mandarim. 
Para a obtenção dos dados, foram selecionadas dez expressões idiomáticas com as palavras mãos, pés e dedos, com o objetivo de manter uma relação semântica entre as mesmas, conforme apresentado no Quadro 1.

\section{Quadro 1 - Expressões idiomáticas}

\begin{tabular}{|l|l|}
\hline Ficar no pé & Ter mão leve \\
Estar com as mãos atadas & Ganhar (dar) de mão beijada \\
Meter os pés pelas mãos & Passar a mão na cabeça \\
Ficar cheio de dedos & Não ter pé nem cabeça \\
Dar a mão à palmatória & Ser uma mão na roda \\
\hline
\end{tabular}

Além da escolha das EIs, foram selecionados textos da internet que as contemplassem. Os textos são todos autênticos, mas a maioria deles foi editada - em geral, optou-se por uma versão mais curta do texto original e buscou-se apresentá-los aos participantes com o layout mais próximo do original. Como ilustração, o conteúdo de dois desses textos está transcrito a seguir. O primeiro, intitulado "Os pinguinhos da reforma", é um editorial do jornalista Jayme Copstein disponível em seu blog, e o segundo, "Atube catcher", um tutorial sobre um software que facilita o download de vídeos. Ambos são uma versão reduzida do original.

\section{Primeiro texto}

Entrevista do escritor, poeta e crítico literário Ivan Junqueira anteontem, no Programa do Jô, trouxe à baila a recente reforma ortográfica que extinguiu algumas miuçalhas a que não se dava a menor importância, acabou com o trema que não fazia mal a ninguém e, de positivo, apenas reconheceu usucapião de lugar no alfabeto das letras "k", "y" e "w", as quais já estavam aí desde sempre. Junqueira, afora ser um dos melhores tradutores de literatura estrangeira, é poeta traduzido até na China. Estava cheio de dedos para falar sobre a reforma, com toda a certeza pensando em não melindrar amigos nela envolvidos. Além de reconhecer a resistência dos portugueses em adotá-la, enfatizou não passarem de pequeninos tantos por cento as mudanças introduzidas.

\section{Segundo texto}

Se você quer salvar vídeos do Youtube ou de outras páginas, precisa conhecer o aTube Catcher. O programa baixa e converte para diversos formatos de maneira simples e rápida. Basta colocar o link no campo "Origem" e fazer o download dos filmes diretamente para a máquina. Com o aTube Catcher tudo é feito no mesmo lugar e sem complicações. O aTube Catcher também serve como conversor de formatos de vídeos. Se quiser os vídeos em formato FLV, por exemplo, basta indicar a posição de saída. Em poucos segundos, você cria uma enorme biblioteca que toca em qualquer player. O programa é uma mão na roda para quem gosta de turbinar e personalizar vídeos. Apesar de a interface poder contundir um pouco no começo, o aTube Catcher é uma excelente solução para baixar, converter e copiar os vídeos favoritos para assistir onde e como quiser.

A coleta de dados ocorreu em sessões individuais, por meio de protocolos verbais de pausa e retrospectivos, que consistem em solicitar que o sujeito verbalize o que está pensando no momento em que busca realizar a tarefa solicitada (protocolo de pausa), e logo após tê-la realizado (protocolo retrospectivo), possibilitando assim que o 
pesquisador obtenha informações sobre os processos cognitivos por ele empregados (ERICSON; SIMON, 1993; AFFLERBACH, 2000; CAMPS, 2003). Todas as verbalizações foram gravadas em áudio, e os dados, posteriormente transcritos.

As seguintes etapas foram adotadas durante a aplicação dos instrumentos: (i) apresentação das EIs descontextualizadas aos sujeitos, solicitando que procurassem formar um significado para as que julgassem desconhecidas a partir de quaisquer tipos de conhecimento que possuíssem; (ii) leitura dos textos que continham as EIs consideradas desconhecidas, convidando-os a explicarem o significado atribuído à expressão após a leitura, como também a relatarem em que medida ele poderia ser comparado ao atribuído à expressão descontextualizada. (iii) realização de protocolos retrospectivos, com a intenção de promover uma reflexão sobre aspectos específicos do processo inferencial do sujeito, com base em questões lançadas pelo pesquisador.

\section{ANÁLISE DOS DADOS}

\subsection{ESTRATÉGIAS EMPREGADAS NAS EIS DESCONTEXTUALIZADAS}

A partir das informações disponibilizadas pelos participantes, foi possível observar o emprego de três estratégias durante a tentativa de realização das inferências das EIs quando descontextualizadas:(i) analogias/associações, a partir de conhecimentos prévios gerais; (ii) repetição ou paráfrase da EI; (iii) relação com EIs na L2 ou na L1. Houve ainda situações em que os participantes, frente a determinadas EIs, refletiam por alguns segundos e afirmavam não conseguir atribuir qualquer significado à expressão. Elas estão classificadas, na Tabela 1, como "desistência de tentativa".

Entre as três estratégias utilizadas pelos participantes, a mais frequente foi a realização de analogias/associações derivadas de conhecimentos prévios, conforme apresentado na Tabela 1. Esse dado está em conformidade com os achados de Sadeghi, Dastjerdi e Ketabi (2010, p. 87) sobre o emprego de estratégias inferenciais para a realização de EIs na L2 pelos participantes de seu estudo, entre as quais figura o uso de imagens metafóricas (e/ou fantásticas).

\section{Tabela 1 - Estratégias para inferências de Els descontextualizadas}

Um exemplo dessas associações está na justificativa do participante 2 à resposta relativa ao significado da EI "cheio de dedos". A sua ideia de que esta significaria ter muita vontade, estar muito empolgado para fazer algo adveio da seguinte associação: quanto mais dedos em uma mão, mais rápida e eficientemente uma tarefa pode ser realizada. Outro exemplo de uso dessa estratégia encontra-se no protocolo do 
participante 4, quando define a mesma EI como "ter mais tarefas do que seria possível abarcar", a partir da comparação estabelecida entre dedos em demasia e tentáculos (em demasia) do polvo. Ainda um terceiro exemplo que ilustra a diversidade das relações criadas pelos aprendizes está na resposta do Sujeito 5: ficar cheio de dedos seria ficar cheio de ideias e ter muitos recursos para resolver determinadas situações, já que "como a mão é um instrumento fundamental para resolver as coisas, então ter mais dedos significa ter mais formas de resolver" (Excerto do Protocolo Verbal do Sujeito 5)

Como exemplos finais, transcrevemos a seguir trechos dos protocolos dos Sujeitos 4 e 5, respectivamente, ao buscarem atribuir significado à EI "ser uma mão na roda", quando apresentada sem contexto. ${ }^{4}$

\section{Protocolo Sujeito 4}

S4: Ser uma mão na roda...Eu acho que é quando uma pessoa vai obstruir, vai atrapalhar alguma coisa...é...uma mão na roda.

P.: No sentido de atrapalhar?

S4: Sim. Tipo, eu imagino, vem uma roda rodando, e você mete a mão. Como eu poderia explicar essa expressão em uma analogia, em um evento? Quando alguém faz alguma coisa de propósito, para atrapalhar ou.... Como é que eu... Não é normal alguém botar a mão na roda, não? Como é que eu... Qual é a proposta de alguém botar a mão na roda? É se ferir ou atrapalhar o andamento da roda.

P.: Sim, entendo.

\section{Protocolo Sujeito 5}

S5: Ser uma mão na roda...(ri)... Eu não sei como fica...

P.: A gente diz assim, alguém ou algo é uma mão na roda.

S5: Eu não sei...eu não ouvi...

P.: E assim, olhando para a expressão, para a palavra roda, te arriscarias em pensar em algum significado?

S5: Não sei, de repente uma pessoa foi útil para outra em uma tarefa.

P.: E porque essa ideia de útil?

S5: Não sei, uma mão na roda....não sei por quê.

P.: Porque só olhando para a expressão, ela não te dá por si só a ideia de ser útil. Deves ter feito alguma relação, não?

S5: É, eu não sei, porque a roda que eu imaginei é a roda feita por pessoas, então a mão na roda faz parte da roda.

P.: Como seria isso?...Ah, uma roda de crianças, uma roda de pessoas... sim, sim, agora entendi.

Dois fatos nos parecem merecedores de atenção nas transcrições precedentes, por ilustrarem a complexidade e diversidade de caminhos cognitivos trilhados por diferentes sujeitos na realização de inferências lexicais. O primeiro refere-se aos significados inferidos para a mesma EI - ou seja, em oposição direta um ao outro. O segundo reside no fato de que, embora o contexto criado para compreender a EI descontextualizada

\footnotetext{
${ }^{4}$ As falas do pesquisador são indicadas pela inicial $\mathrm{P}$, e do sujeito, por S. O uso de reticências nas transcrições indica uma pequena pausa no fluxo do discurso dos sujeitos, nunca maiores do que, aproximadamente, oito segundos. Pausas maiores são interrompidas pelo pesquisador, a fim de que o sujeito continue a verbalizar seu pensamento.
} 
pelo participante 5 não confira com o contexto original utilizado em português para explicá-la, o significado inferido está de acordo com o usualmente conhecido em língua portuguesa - ou seja, ser um mão na roda é ser útil, auxiliar algo e/ou alguém.

Com relação à segunda estratégia de uso mais frequente, repetição da expressão, reiteramos aqui o que já afirmamos em outro trabalho (BALDO, 2006, p. 159): trata-se de um recurso cognitivo utilizado para reter na memória a EI cujo significado está sendo buscado através de todos as fontes de conhecimento disponíveis, sejam elas de conhecimento enciclopédico, sejam elas de conhecimento da L1 e/ou da L2. Além disso, é importante ressaltar que a repetição era acompanhada, na maioria das vezes, pela dificuldade e posterior desistência de encontrar um significado para a EI, mas não em todos os casos. Como ilustração, tem-se a reação do Sujeito 5 frente à EI "meter os pés pelas mãos". Após pensar por alguns segundos, repetir a expressão em voz alta e voltar a refletir novamente, o participante sugeriu como significado "muita confusão, atrapalhado". Nesse sentido, cabe relembrar o estudo de Nassaji (2003), no qual o fato de a repetição ser a estratégia mais empregada para a realização de inferências lexicais não ter sido considerado surpreendente pelo autor na medida em que ela auxilia na compreensão e na reflexão sobre o conteúdo.

Sobre o uso do background linguístico através da relação com outras EIs, tanto na L1 como na L2, o dado que mais chama a atenção - mas também é o mais simples de explicar - é seu emprego modesto, de apenas 4 ocorrências, em comparação com a estratégia 1. A explicação está na natureza das EIs selecionadas para o estudo: entre as dez EIs, apenas três possuem equivalentes idênticos em espanhol, a L1 de três dos participantes, e nenhuma delas na L1 dos outros dois. Isso representa, naturalmente, um obstáculo maior para o emprego da estratégia, e está em conformidade com achados de pesquisa recentes que aproximam o grau de opacidade da EI na L2 com sua equivalente na L1 ao nível de dificuldade de processamento pelo aprendiz de L2 (LIONTAS, 2002; BULUT; ÇELIK-YAZICI, 2004; SADEGHI; DASTJERDI; KETABI, 2010). Corroborando essa ideia está o fato de que as relações entre outras EIs na L1 ou na L2 foram estratégias empregadas somente pelos falantes de espanhol como L1. A única relação estabelecida com outra EI na L1 também foi feita por um falante de espanhol como L2, o Sujeito 5. A seguir estão transcritos os trechos dos protocolos do Sujeito 4, ao equiparar a EI na L2 com a L1, e o do Sujeito 5, ao utilizar o conhecimento de uma EI da L1 para interpretar a nova EI "ficar no pé".

\footnotetext{
S4: Eu acho que isso já sei é igual como no espanhol [...] quer dizer, quando você fala alguma coisa que não tem nada a ver, saiu do contexto totalmente.

S5: Eu não sei, só sei que "tal coisa ainda tá em pé", é tal coisa ainda tá valendo, não sei se pode ser...

P.: Não, não é a mesma, é uma outra expressão. Essa expressão, ficar no pé, não te remete a alguma outra ideia?

S5: Não sei, o que eu pensei foi o sentido daquela outra expressão...

P.: Estar de pé. E achas que pode ter um sentido parecido com essa?

S5: (pensa um pouco)... Não sei, acho que sim.
} 
Ainda outro aspecto a considerar com relação ao resgate de EIs similares na L1 para a inferência de EIs na L2 foi o uso relativamente limitado entre os falantes de espanhol. Se considerarmos que havia três EIs dessa natureza e três sujeitos cuja L1 era o espanhol, havia condições para um número maior de ocorrências. Nesse sentido, esse dado poderia ser interpretado como uma confirmação do achado de Bulut e Çelik-Yazici (2004) de que alguns participantes de seu estudo, ainda que não a maioria deles, tiveram receio de tratar-se de falsos cognatos e preferiram empregar o contexto para compreender EIs na L2 com equivalentes na L1.Contudo, trata-se somente de uma especulação, considerando que os dados obtidos aqui não possibilitam afirmações dessa natureza.

\subsection{ESTRATÉGIAS EMPREGADAS NAS EIS CONTEXTUALIZADAS}

As seguintes estratégias foram verificadas durante a tarefa de inferência do significado das EIs em contexto: (i) uso de indícios micro e /ou macrotextuais disponibilizados pelo texto; (ii) releitura da frase que contém a expressão; (iii) solicitação de informação sobre a EI - em alguns casos, a fim de obter aprovação, pelo pesquisador, do sentido inferido; (iv) relação com outra expressão na L1.

\section{Tabela 2 - Estratégias para inferências de Els contextualizadas}

\begin{tabular}{lr}
\hline Estratégias & Ocorrências \\
\hline Uso de indícios micro e/ou macrotextuais & 22 \\
Releitura da frase que contém a expressão & 10 \\
Solicitação de informação sobre a EI & 7 \\
(aguardo da confirmação da inferência feita) & 1 \\
Relação com outra EI na L2 & \\
\hline
\end{tabular}

A exemplo da situação apresentada pelos sujeitos com relação às EIs descontextualizadas, aqui também apenas uma estratégia aparece com significativa frequência de uso, ou seja, uso de indícios micro e/ou macrotextuais. Esse resultado encontra respaldo nos estudos citados na seção precedente, cujas análises revelaram, sem exceção, o papel-chave do contexto para a inferência de EIs na L2 (COOPER, 2009; LIONTAS, 2002; BULUT; ÇELIK-YAZICI， 2004; POLÓNIA， 2009; SADEGHI, DASTJERDI; KETABI, 2010).

Mostramos seu uso por dois exemplos do Sujeito 5, o primeiro quando justifica o significado encontrado para a EI "ficar no pé", e o segundo, quando explica como entendeu a expressão "dar a mão à palmatória".

S5: Eu não sei se significa mais ou menos...no sentido de ficar mais atenta, chamar à atenção, insistir.

P.: O que te faz pensar nisso?

S5: Porque o texto diz que não é infrequente encontrar crianças que não costumam comer alimentos saudáveis, e da situação, que esse costume das crianças tem a ver com as mães, e 
como elas educam, e quando as crianças não querem comer "nada melhor que as mamães para ficar no pé dos baixinhos".

S5: Acho que é no sentido de ser humilde, aceitar a decisão dos outros.

P.: O que te levou a pensar nesse significado?

S5: É porque ele estava dizendo "tenha ideias próprias e saiba dar a mão à palmatória"

Acho que é nesse sentido então, quando os outros têm ideias interessantes e pertinentes, precisamos ser mais humildes, de aceitar.

Como justificativa para a segunda estratégia mais usada, releitura da frase que contém a expressão, com 10 ocorrências, a conclusão de Nassaji (2003), já citada na seção anterior, continua válida: a repetição - aqui, via releitura - auxilia na compreensão e na reflexão sobre o conteúdo sob apreciação. Além disso, cabe lembrar aqui a hipótese de Kern (1989) sobre os traços mais fracos de memória no processamento na L2, na medida em que a necessidade de voltar ao texto poderia sugerir um apagamento das informações lidas. Nesse sentido, a releitura parcial do texto auxiliaria a fixar na memória os dados necessários para a realização da inferência das EIs. ${ }^{5}$

Por fim, informações foram solicitadas ao pesquisador pelos participantes com dois objetivos: obter (i) o significado de palavras integrantes das EIs ou das sentenças próximas a elas no texto e (ii) certificar-se de que o significado inferido estava apropriado. Tanto um como outro caso parecem evidenciar, simplesmente, as restrições impostas pelo novo sistema linguístico em construção - ou, em outras palavras, impostas pela interlíngua dos aprendizes.

Esse mesmo motivo parece ser a explicação mais plausível para o fato de somente um participante, em apenas uma ocasião, ter resgatado o significado de uma EI na L2 ao tentar inferir a nova EI - ou seja, "estar em cima" para definir "ficar no pé", conforme transcrição a seguir.

S2: Pelo contexto, as mães, para ficar atentas aos seus filhos, ficar...Não sei qual é....Como estar atento, cuidar-lhes...

P: É nessa direção. Por exemplo, uma mãe que está sempre insistindo que o filho coma frutas, legumes, etc.

S2: Sim, como "estar em cima".

\subsection{ESTRATÉGIAS EM COMUM:} EIS DESCONTEXTUALIZADAS E CONTEXTUALIZADAS

Com relação ao terceiro objetivo do estudo, ou seja, o de avaliar em que medida seria possível aproximar os processos cognitivos empregados pelos sujeitos na inferência das EIs descontextualizadas e contextualizadas, os dados encontrados nos mostram que a aproximação pode ser somente parcial.

\footnotetext{
5 Ainda que o desenho do estudo não possibilite verificar essa hipótese, a citamos aqui não somente porque a consideramos procedente, mas também porque desejamos chamar a atenção para a relevância de pesquisas que busquem investigá-la.
} 
Duas estratégias têm uso comum nos dois contextos: relação com outras EIs, tanto na L1 como na L2, e repetição/releitura. Ainda que repetição e releitura sejam atividades cognitivas diferentes, elas são consideradas equivalentes neste estudo na medida em que, devido à própria metodologia adotada, na primeira atividade proposta (com EIs descontextualizadas), a repetição "substitui" a releitura. De fato, os participantes por vezes reliam a EI em voz alta mesmo quando esta era o único pedaço de informação que constava na folha de papel a sua frente, durante a coleta de dados. Para fins de análise, classificamos essas ocorrências como "repetição", mas entendemos que "releitura" seria igualmente aceitável. Esses dados são apresentados na Tabela 3.

\section{Tabela 3 - Estratégias em comum entre dois contextos de Els}

\begin{tabular}{lrrr}
\hline EIs & Relação com EI na L1 & Relação com EI na L2 & Repetição (releitura) \\
EIs descontextualizadas & 1 & 2 & 5 \\
EIs contextualizadas & 3 & 1 & 10 \\
\hline
\end{tabular}

A seguir, dois exemplos do uso de relação com outras EIs na L2 são apresentados. No primeiro, o participante inferia a EI desprovida de contexto, enquanto no segundo a inferência se deu a partir da leitura de um texto.

Expressão descontextualizada: dar de mão beijada.

S2: Pode ser ganhar algo sem fazer muito esforço?

P.: Sim. Como tu chegaste a isso?

S2: Acho que no espanhol tem algo assim.

Expressão contextualizada: cheio de dedos.

S1: Cheio de dedos... mas ...(pensa em silêncio)...não, não...

P: Ele deu uma entrevista falando sobre a reforma, não?

S1: Sim, entendo.

P: E tinha amigos que faziam parte dessa reforma...

S1: Não seria como atado de pé à cabeça, ou atado de manos?..

Dos dois exemplos de ocorrências de relação com EIs na L1, um já foi apresentado na seção anterior (relação entre EIs "ficar no pé" e "estar de pé"; do Sujeito 5). O outro é apresentado a seguir, pelo protocolo verbal do Sujeito 4 ao estabelecer relação entre as EIs "meter os pés pelas mãos" e "estar com a cabeça quente".

S4: Eu li que quando estamos sob determinado estresse, a gente faz coisas que não deveria fazer. Aí ele utiliza o termo meter os pés pelas mãos quando a pessoa vai dizer o que não deve, discute, ou seja, a pessoa faz as coisas com a cabeça quente.

Cabe frisar, contudo, que, como mostrado na Tabela 3, a tentativa de relacionar a EI na língua-alvo com outra EI, seja no contexto da L1, seja no contexto da L2, foi feita 
poucas vezes, pelas razões já mencionadas anteriormente. Já a estratégia de repetição/releitura foi utilizada de modo um pouco mais significativo, sobretudo quando as EIs estavam contextualizadas, mas ainda assim com ocorrências bem mais baixas do que as duas estratégias de maior frequência em cada um dos contextos.

Essas estratégias - ou seja, analogias ou associações a partir de conhecimentos prévios gerais para inferências das EIs descontextualizadas, e uso de indícios textuais para inferências de EIs em contexto - possuem, claramente, natureza diversa. No primeiro caso, tem-se uso de um contexto amplo, variável de participante para participante, que vai desde associações por imagens até experiências prévias de vida. No segundo, os processos inferenciais são guiados - e limitados - pelo contexto apresentado pelo texto, e a variação fica restrita ao tipo de indício selecionado pelo leitor, macro ou microtextual. E é por isso que reiteramos que a aproximação encontrada entre os dois processos inferenciais é - e sempre deverá ser - apenas parcial.

\section{CONSIDERAÇÕES FINAIS}

Aos resultados deste estudo, cabem duas ressalvas. A primeira delas refere-se à impossibilidade de serem generalizados, dada a natureza qualitativa da pesquisa. A contribuição, até o momento, reside na corroboração - e, por vezes, problematização de achados de pesquisas prévias sobre o tema, conforme procedido ao longo do texto.

A segunda, ao fato de serem relativos à primeira parte de um estudo que prevê continuidade. A finalização desta etapa será seguida pela: (i) aplicação dos instrumentos a um número maior de aprendizes de português como L2, para verificar se o mesmo padrão de uso de estratégias encontrado até o momento será confirmado; (ii) apresentação de novas EIs para os sujeitos, a fim de verificar se a natureza das expressões - tanto com base em metáforas primárias e com relação a campos semânticos específicos - atua como uma variável na seleção das estratégias empregadas no processo inferencial.

Retomando brevemente o conteúdo da seção anterior, a análise dos protocolos mostrou que: (i) a estratégia mais utilizada para inferência das EIs descontextualizadas foi a associação e analogia a partir de conhecimentos prévios gerais, enquanto o uso de indícios textuais foi a escolha preferencial para as EIs em contexto; (ii) o grau de opacidade da EI na L2 com sua equivalente na L1 tem relação direta com a dificuldade de processamento da expressão pelo aprendiz de L2; (iii) a repetição e releitura - esta especificamente no caso das EIs contextualizadas - também foram empregadas, e seu uso é justificado na medida em que podem auxiliar a compreensão e reflexão do conteúdo analisado; (iv) os participantes usaram duas estratégias comuns para a inferência das EIs descontextualizadas e contextualizadas, o que levou à conclusão de que há uma aproximação parcial entre os processos inferenciais em cada um dos contextos; (v) os dados sugerem que essa aproximação sempre terá um caráter relativo, considerando-se as especificidades de cada um dos contextos inferenciais.

Esperamos que as evidências apresentadas, mesmo que parciais, tenham sido suficientes para mostrar a complexidade dos recursos inferenciais de que os aprendizes 
deste estudo lançaram mão na tentativa de buscarem sentido para expressões que, em um primeiro olhar, não lhes diziam nada. Esperamos também que este estudo sirva de estímulo para que profissionais envolvidos com o ensino-aprendizagem de L2 lancem um olhar mais atento aos métodos e técnicas de ensino de vocabulário - e, especificamente, de EIs - que têm empregado em sua prática docente. Como apontado por este e outros estudos, cada aprendiz de L2 parece percorrer um caminho único no seu esforço para atribuir significados a palavras e expressões desconhecidas, e esse dado não pode ser ignorado na aula de língua estrangeira.

\section{REFERÊNCIAS}

AFFLERBACH, P. Verbal reports and protocol analysis. In: PEARSON, D. P.; KAMIL, M. L.;

MOSENTHAL, P. B.; BARR, R. (Orgs.). Handbook of reading research III. Londres: Routledge, 2000, p. 163-175.

BALDO, A. Uso de estratégias de leitura na língua materna e na língua estrangeira. 2006. 198f. Tese (Doutorado em Letras). Porto Alegre, PUCRS, 2006.

BULUT, T.; ÇELIK-YAZICI, I. Idiom processing in L2: through rose-colored glasses. The Reading Matrix, v. 4, n.2, p.105-116, 2004

CAMPS, J. Concurrent and retrospective verbal protocols as tools to better understand the role of attention in second language tasks. International Journal of Applied Linguistics, v. 13, n.2, p. 201-221, 2003.

COOPER, T. C. Processing of idioms by L2 learners of English. TESOL Quarterly, v. 33, n. 2, p. 233 $262,1999$.

CORNEL, A. An approach to identifying major pitfalls for learners. IRAL, v. 37, n. 1, p.1-11, 1999.

ERICSON, K.A; SIMON, H. Protocol analysis: verbal report as data. MIT Press, Cambridge, MA, 1993.

GIBBS, R. The poetics of mind: figurative thought, language, and understanding. Cambridge: Cambridge University Press, 1994.

KERN, R. Second language reading strategy instruction: its effects on comprehension and word inference ability. The Modern Language Journal, v. 73, n. 2, p. 135-149, 1989.

KRAMSCH, C. Context and culture in language teaching. Oxford: Oxford University Press, 1993.

LAKOFF, G.; JOHNSON, M. Metaphors we live by. London: The University of Chicago Press, 1980.

LIONTAS, J. I. That's all Greek to me! The comprehension and interpretations of Modern Greek idioms. Reading Matrix, vol. 1, n.1, p.1-32, 2001.

NASSAJI, H. L2 vocabulary learning from context: strategies, knowledge sources and their relationship with success in L2 lexical inferencing. TESOL Quarterly, v. 37, n.4, p. 645-670, 2003.

ORTIZ ALVAREZ, M. L. Expressões idiomáticas do português do Brasil e do espanhol de Cuba: estudo contrastivo e implicações para o ensino de português como língua estrangeira. 2000. 334f. Tese (Doutorado em Linguística). Universidade Estadual de Campinas, Campinas, 2000.

As expressões idiomáticas nas aulas de ELE: um bicho de sete cabeças? In: REY, I. G.(org.) Les expressions figées em didactique des langues étrangères. 1. ed. Proximités E.M.E, 2007, p.159-179 .

; MACHADO, A. E. B. Expressões idiomáticas em textos jornalísticos: insumo para as aulas de PLE. Horizontes de Linguística Aplicada, v. 9, n. 2, p.145-162, 2010.

PARANÁ, J. M. F.; ALMEIDA, M. R. Seminários sobre interculturalidade - em busca do resgate da cultura no ensino de língua estrangeira. In: GIMENEZ, T.; JORDÃO, C. M.; ANDREOTTI, V. (Orgs.). Perspectivas educacionais e o ensino de inglês na escola pública. Pelotas: Educat, 2005, p. 69-88.

PEDRO, M. L. As expressões idiomáticas no ensino de português como língua estrangeira para estudantes uruguaios. 2007. 197f. Dissertação (Mestrado em Linguística). Universidade de Brasília, Brasília (DF), 2007. 
POLÓNIA, C. P. F. M. As expressões idiomáticas em português língua estrangeira: uma experiência metodológica. Dissertação (Mestrado em Letras). Porto: Universidade do Porto, 1999.

RIVA, H. C.; CAMACHO, B. F. Expressão idiomática: uma unidade fraseológica. In: BARROS, L. A.; ISQUIERDO, A. N. (Orgs.). O léxico em foco: múltiplos olhares. São Paulo: Editora UNESP, 2010, p. 195-218

SADEGHI, B.; DASTJERDI, H.; KETABI, S. Patterns of Persian EFL learners' comprehension of idiomatic expressions: reading strategies. Canadian Center of Science and Education, v. 6, n.8, p. 81-99, 2010.

SILVA, N. H. Metáfora e metonímia nas construções com pé: uma abordagem cognitivista. 2011. 112f. Dissertação (Mestrado em Letras). Universidade Federal do Rio de Janeiro, Rio de Janeiro, 2011.

TAGNIN, S. E. O. O jeito que a gente diz: expressões convencionais e idiomáticas. São Paulo: Editora Disal, 2005.

\section{Recebido em 02/10/13. Aprovado em: 05/08/14.}

Title: Understanding Portuguese idiomatic expressions as L2: evidence of verbal protocols

Author: Alessandra Baldo

Abstract: A report is presented on a study that aimed at investigating to what extent the cognitive processes used by five Portuguese L2 speakers to understand ten idiomatic phrases (EIs) in a decontextualized way would be similar to those used for the understanding of the same phrases in a context. The analysis of the data, collected by means of retrospective verbal protocols and protocols of pause, revealed that the use of analogy and associations based on previous knowledge was the preferential strategy for the decontextualized idiomatic phrases, while the use of microtextual signs was the most used one in those EIs on a context. In addition, the data pointed to an approximation only partial of the cognitive processes used by the participants in both contexts of attribution of meaning to new EIs. The results are assessed based on Liontas (2001, 2002), Polónia (2009) and Sadeghi, Dastjerd and Ketabi (2010).

Keywords:Idiomatic expressions. Iinferential processes. Portuguese as L2.

Título: Comprensión de Expresiones Idiomáticas de la Lengua Portuguesa como L2: evidencias de protocolos verbales

Autora: Alessandra Baldo

Resumen: Este artículo expone un estudio cuyo objetivo fue investigar en qué medida los procesos cognitivos utilizados por cinco hablantes de portugués como L2 para comprender diez expresiones idiomáticas (EIs) de manera descontextualizada podrían ser similares a aquellos utilizados para la comprensión de las mismas expresiones en contexto. El análisis de datos, recogidos por medio de protocolos verbales retrospectivos y de pausa, reveló que el uso de analogía y asociaciones desde conocimientos previos fue la estrategia preferencial para las EIs descontextualizadas, mientras el uso de indicios micro textuales fue la más empleada en las EIs en contexto. Además, los datos apuntaran para una aproximación solamente parcial de los procesos cognitivos empleados por los participantes en los dos contextos de atribución de significado para nuevas EIs. Los resultados son evaluados con base en Liontas (2001, 2002), Polónia (2009) e Sadeghi, Dastjerd e Ketabi (2010).

Palabras-clave: Expresiones idiomáticas. Procesos inferenciales. Portugués como L2. 